\title{
Downregulation of miR-588 is associated with tumor progression and unfavorable prognosis in patients with osteosarcoma
}

\author{
TAO YU ${ }^{1}$, SHUANG LIANG ${ }^{2}$, TIANHONG MA ${ }^{2}$ and WEIDONG SONG ${ }^{1}$ \\ ${ }^{1}$ Department of Orthopedics, The Second Affiliated Hospital of Mudanjiang University; ${ }^{2}$ Department of Radiology, \\ Hongqi Hospital Affiliated to Mudanjiang Medical College, Mudanjiang, Heilongjiang 157009, P.R China
}

Received October 30, 2020; Accepted March 8, 2021

DOI: $10.3892 /$ etm.2021.10024

\begin{abstract}
Osteosarcoma (OS) is a primary malignant tumor characterized by a high metastatic potential and poor prognosis. The dysregulation of miR-588 has been demonstrated to serve crucial roles in the progression of numerous types of cancer. The present study aimed to investigate the expression and function of miR-588 in the development of OS. To do so, clinical samples were collected and analyzed, and in vitro experiments were conducted. A total of 104 patients with OS were recruited between 2012 and 2014. The expression of miR-588 was analyzed by reverse transcription quantitative PCR. The association between miR-588 expression and the clinicopathological characteristics and survival rate of patients with OS was evaluated. Furthermore, Cell Counting Kit-8 and Transwell assays were used to evaluate the effect of miR-588 on the proliferation and the migratory and invasive abilities of various OS cell lines. The results demonstrated that miR-588 expression in OS tissues and cells was significantly lower compared with normal tissues and cells. In addition, miR-588 expression was closely associated with the Musculoskeletal Tumor Society (MSTS) staging of patients with OS. miR-588 expression and MSTS staging were therefore considered as independent indicators for the prognosis of patients with OS. In addition, miR-588 downregulation significantly stimulated the proliferation and migratory and invasive abilities of OS cells. Taken together, these findings indicated that miR-588 may serve as an independent prognostic factor and tumor suppressor in OS.
\end{abstract}

\section{Introduction}

Osteosarcoma (OS) is the most frequent primary solid malignancy of the bone characterized by the presence of malignant

Correspondence to: Dr Weidong Song, Department of Orthopedics, The Second Affiliated Hospital of Mudanjiang University, 15 Dongxiaoyun Street, Mudanjiang, Heilongjiang 157009, P.R. China

E-mail: songwddoc@163.com

Key words: osteosarcoma, miR-588, prognosis, tumor progression, development mesenchymal cells producing osteoid or immature bone (1). The incidence of OS is estimated at 5 per million persons, accounting for $45.3 \%$ of bone tumors worldwide $(2,3)$. The alterations in the expression of the corresponding genes that encode tumor suppressors and promotors are closely associated with the incidence of OS. In the last decades, the therapeutic strategies for OS have rapidly improved, including T-cell related, occupational and physical therapy $(4,5)$; however, the prognosis of patients with OS remains poor due to the high rates of tumor metastasis and recurrence (6-9). At present, the identification of new therapeutic targets that are associated with the occurrence, development and metastasis of cancers has drawn special attention, which may provide novel approaches for the clinical management and treatment of OS (10).

MicroRNAs (miRNAs) are a class of non-coding RNAs that have been reported to regulate the expression of target genes by binding to the 3'-untranslated region of target mRNAs (11). Furthermore, miRNAs have been demonstrated to serve crucial roles in tumor cell development, differentiation, proliferation and apoptosis. Subsequently, various miRNAs have been identified as regulators in the biogenesis and progression of certain types of cancer, such as miR-154 in gastric cancer, miR-133a in non-small cell lung cancer, miR-19b in breast cancer and miR-769 in colorectal cancer (12-15). Numerous aberrantly expressed miRNAs were reported to participate in the development and prognosis of OS. For example, it was demonstrated that downregulated miR-223-3p can predict the poor prognosis of patients with OS and that miR-223-3p can inhibit OS cell proliferation, invasion and migration by regulating the expression of cadherin 6 (16). Furthermore, miR-552-5p was reported to serve as a tumor promoter that could facilitate the development and progression of OS (17). A previous study demonstrated that miR-588 is downregulated in breast cancer and that it can inhibit the proliferation of breast cancer cells (18). The dysregulation and functional role of miR-588 in tumor cell development was observed in prostate cancer, gastric cancer and lung squamous cell carcinoma (19-21). A previous study reported that miR-588 is downregulated in OS (22); however, its function and specific role in the progression of OS and the prognosis of patients remain unclear.

The present study aimed to evaluate the expression and biological function of miR-588 in OS tissues and cells and to determine the prognostic value of miR-588 in patients with OS. 


\section{Materials and methods}

Tissues and cell lines. This study was approved by the Ethics Committee of The Second Affiliated Hospital of Mudanjiang University (approval no. 200934; Mudanjiang, China). A total of 104 patients diagnosed with OS at The Second Affiliated Hospital of Mudanjiang University were recruited between October 2010 and November 2014. The inclusion criteria for patients with OS were as follows: i) Patients were diagnosed with OS as a primary malignancy; ii) none of the patients had received any anti-tumor therapy, radiotherapy or chemotherapy; iii) all patients signed written informed consent and participated in a 5-year follow-up survey; and iv) the clinicopathological characteristics of patients with OS was provided. Patients with a history of other cancers were excluded from this study. Paired OS tissues and adjacent normal tissues were collected during resection surgery and confirmed by at least two pathologists. Tissues were immediately frozen in liquid nitrogen and stored at $-80^{\circ} \mathrm{C}$ prior to following experiments.

The OS cell lines MG63, U2OS, KHOS-240S and SAOS-2, and the human osteoblast cell line hFOB 1.19 were purchased from the American Type Culture Collection. All cells were cultured in DMEM medium (Thermo Fisher Scientific, Inc.) supplemented with 10\% FBS (Thermo Fisher Scientific, Inc.) and placed at $37^{\circ} \mathrm{C}$ in a humidified incubator containing $5 \% \mathrm{CO}_{2}$.

Cell transfection. In order to regulate the expression of miR-588 in OS cells, miR-588 mimic $(50 \mathrm{nM}$; 5'-UUG GCCACAAUGGGUUAGAAC-3'), miR-588 inhibitor (50 nM; 5'-GUUCUAACCCAUUGUGGCCAA-3'), mimic negative control (mimic NC; $50 \mathrm{nM}$; 5'-UUCUCCGAACGUGUCACG UU-3') and inhibitor negative control (inhibitor NC; $50 \mathrm{nM}$, 5'-UUGCUAGUGCGACACUACUUCTT-3') (all from Shanghai GenePharma Co., Ltd.) were transfected into OS cells using Lipofectamine $2000^{\mathrm{TM}}$ reagent (Invitrogen; Thermo Fisher Scientific, Inc.) according to the manufacturer's instructions. The transfection mix was prepared at room temperature and cells were incubated with the transfection complexes at $37^{\circ} \mathrm{C}$ for $4 \mathrm{~h}$. Transfected cells were subjected to subsequent experiments after $48 \mathrm{~h}$ of transfection. Non-transfected cells were used as the mock group.

$R N A$ extraction and reverse transcription quantitative (RT- $q$ ) $P C R$. Total RNA from tissues and cells was extracted using TRIzol $^{\circledR}$ reagent (Invitrogen; Thermo Fisher Scientific, Inc.). Following reverse transcription to cDNA using the TaqMan MicroRNA Reverse Transcription kit (Invitrogen; Thermo Fisher Scientific, Inc.), RT-qPCR was used to determine the expression of miR-588 with the SYBR Green I Master Mix kit (Invitrogen; Thermo Fisher Scientific, Inc.). The sequences of the primers were as follows: miR-588, forward 5'-GGGUUG GCCACAAUGGGU-3', reverse 5'-GTCGTATCCAGTGCG TGT-3'; and U6, forward 5'-CTCGCTTCGGCAGCACA-3' and reverse 5'-AACGCTTCACGAATTTGCGT-3'. The thermocycling conditions were as follows: $95^{\circ} \mathrm{C}$ for $30 \mathrm{sec}$, followed by 40 cycles of annealing at $95^{\circ} \mathrm{C}$ for $10 \mathrm{sec}$ and extension at $60^{\circ} \mathrm{C}$ for $30 \mathrm{sec}$. The relative expression levels of miR-588 were normalized to endogenous control U6 and were expressed as $2^{-\Delta \Delta C q}(23)$.
Cell proliferation assay. OS cell proliferation was evaluated with the Cell Counting Kit-8 (CCK-8; Dojindo Molecular Technologies, Inc.) assay. Briefly, $5 \times 10^{3}$ cells were seeded per well into a 96-well plate for $0,24,48$ and $72 \mathrm{~h}$. A total of $10 \mu \mathrm{l} \mathrm{CCK}-8$ was added to each well and cells were incubated for $4 \mathrm{~h}$ at $37^{\circ} \mathrm{C}$ with $5 \% \mathrm{CO}_{2}$. The absorbance was read at $450 \mathrm{~nm}$ using a microplate reader (Beckman Coulter, Inc.). Experiments were performed in triplicate.

Cell migration and invasion assay. Transwell assays were used to detect the migratory and invasive abilities of OS cells. Briefly, $1 \times 10^{4}$ cells were seeded into the upper chamber of a 24-well Transwell chamber (8- $\mu \mathrm{m}$ pore size; Corning, Inc.) and cultured with serum-free medium. The bottom chamber was filled with medium supplemented with $10 \%$ FBS used as a chemoattractant. For the invasion assay, the upper chamber was pre-coated with Matrigel solution (BD Biosciences) at $37^{\circ} \mathrm{C}$ for $1 \mathrm{~h}$ prior to cell seeding. Following $48 \mathrm{~h}$ incubation at $37^{\circ} \mathrm{C}$, the number of cells that have migrated to the bottom chamber were stained with $1 \%$ crystal violet at room temperature for $30 \mathrm{~min}$ and counted using a light microscope (magnification, x200). Experiments were performed in triplicate.

Statistical analysis. Data were expressed as the means \pm standard deviation obtained from at least three independent experiments and were analyzed by SPSS 20.0 software (IBM Corp.) and GraphPad Prism 7.0 software (GraphPad Software, Inc.). The difference between groups was evaluated by paired Student's t-test or one-way ANOVA followed by Tukey's post hoc test. The association between miR-588 expression and the clinicopathological characteristics of patients with OS was analyzed using $\chi^{2}$ test. The overall survival of patients with OS according to their miR-588 expression level was estimated by Kaplan-Meier analysis and log-rank test. The prognostic value of miR-588 in OS was further evaluated by Cox regression analysis. $\mathrm{P}<0.05$ was considered to indicate a statistically significant difference.

\section{Results}

miR-588 is significantly downregulated in OS tissues and cells. The results from RT-qPCR demonstrated that miR-588 expression was significantly decreased in OS tumor tissues compared with adjacent normal tissues $(\mathrm{P}<0.001$; Fig. 1A). Similarly, miR-588 expression was significantly downregulated in OS cells (MG63, KHOS-240S, SAOS-2, and U2OS) compared with hFOB1.19 cells ( $\mathrm{P}<0.001$; Fig. 1B). miR-588 downregulation in OS tissues and cells suggested that it may have a role in the development of OS.

miR-588 downregulation is associated with Musculoskeletal Tumor Society (MSTS) staging of patients with OS. According to the mean expression level of miR-588 in OS tumor tissues (0.384), 104 patients with OS were divided into a low miR-588 expression group $(\mathrm{n}=63)$ and a high miR-588 expression group $(n=41)$. The results demonstrated that miR-588 expression was associated with MSTS staging $(\mathrm{P}=0.033)$ of patients with OS (Table I). No significant association was found between miR-588 expression and the other clinicopathological charac- 
Table I. Association between miR-588 expression and the clinicopathological characteristics of patients with osteosarcoma.

$\operatorname{miR}-588$

$\begin{array}{llll}\text { Characteristics } & \text { Cases }(n=104) & \text { Low expression }(n=63) & \text { High expression }(n=41)\end{array}$

Age, years

$<20$

$\geq 20 \quad 49$

$55-31$

0.633

Sex

Male

60

32

24

$32 \quad 17$

Female

44

35

Female

28

25

Tumor size, $\mathrm{cm}$

$<8$

$\geq 8$

57

30

MSTS staging

$47 \quad 33$

I-II 74

III 30

40

23

0.374

Location

Tibia/femur

68

37

27

14

Elsewhere

36

26

34

7

31

10

MSTS, Musculoskeletal Tumor Society; miR, microRNA.

A

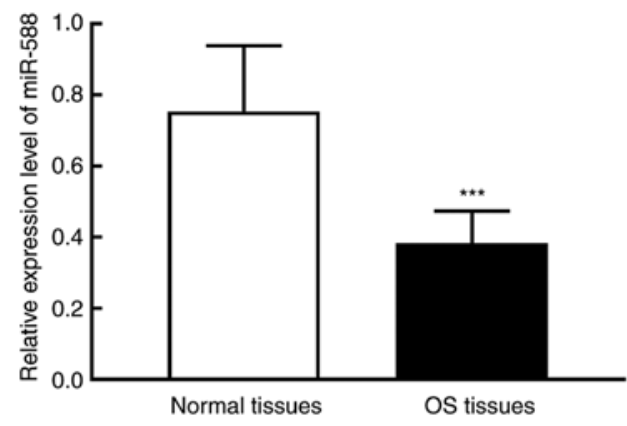

B

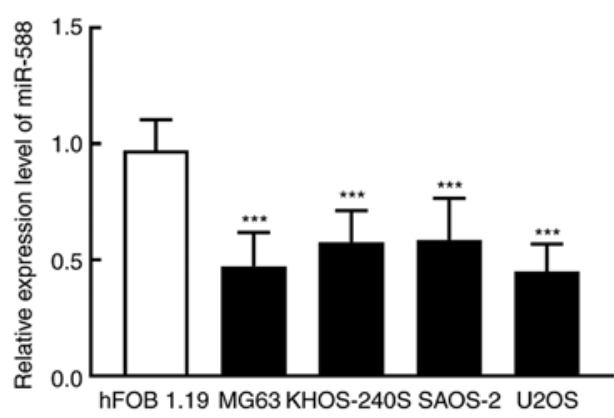

Figure 1. Relative expression of miR-588 in OS tissues and cells. (A) miR-588 was significantly downregulated in OS tissues compared with adjacent normal tissues. ${ }^{* * *} \mathrm{P}<0.001$. (B) miR-588 was significantly downregulated in OS cell lines MG63, HKOS-240S, SAOS-2 and U2OS compared with normal cells hFOB1.19. ${ }^{* * *} \mathrm{P}<0.001$. OS, osteosarcoma; miR, microRNA.

teristics of patients (age, sex, tumor size and location; $\mathrm{P}<0.05$; Table I).

Prognostic value of miR-588 in OS. The Kaplan-Meier curve of patients with OS demonstrated that patients with high

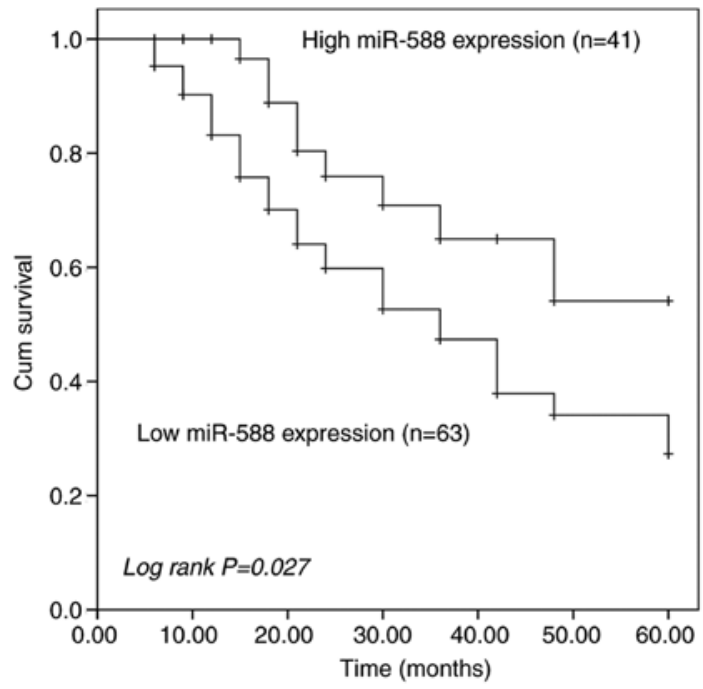

Figure 2. Kaplan-Meier curve of patients with osteosarcoma according to miR-588 expression levels. Log rank $\mathrm{P}=0.027$. miR, microRNA.

miR-588 expression levels had a higher 5-year survival rate than patients with low miR-588 expression levels (log-rank $\mathrm{P}=0.027$; Fig. 2). The prognostic value of miR-588 was further assessed by Cox regression analysis. The results showed that miR-588 expression [hazard ratio $(\mathrm{HR})=2.533$; 95\% confidence interval $(\mathrm{CI})=1.187-5.407 ; \mathrm{P}=0.016]$ and MSTS staging $(\mathrm{HR}=2.517 ; 95 \% \mathrm{CI}=1.088-5.824 ; \mathrm{P}=0.031)$ were independent predictors for the prognosis of patients with OS (Table II).

miR-588 inhibits the proliferation of OS cells. Cell transfections with miR-588 mimic or miR-588 inhibitor were 
Table II. Impacts of clinicopathological characteristics on prognosis in patients with osteosarcoma.

\begin{tabular}{lccr}
\hline Characteristics & HR factor & 95\% CI & P-value \\
\hline miR-588 expression & 2.533 & $1.187-5.407$ & 0.016 \\
Age & 1.195 & $0.625-2.287$ & 0.590 \\
Sex & 1.163 & $0.599-2.258$ & 0.656 \\
Tumor size & 1.353 & $0.686-2.671$ & 0.383 \\
MSTS staging & 2.517 & $1.088-5.824$ & 0.031 \\
Tumor location & 1.745 & $0.853-3.569$ & 0.127
\end{tabular}

MSTS, Musculoskeletal Tumor Society; miR, microRNA; HR, hazard ratio; CI, confidence interval.
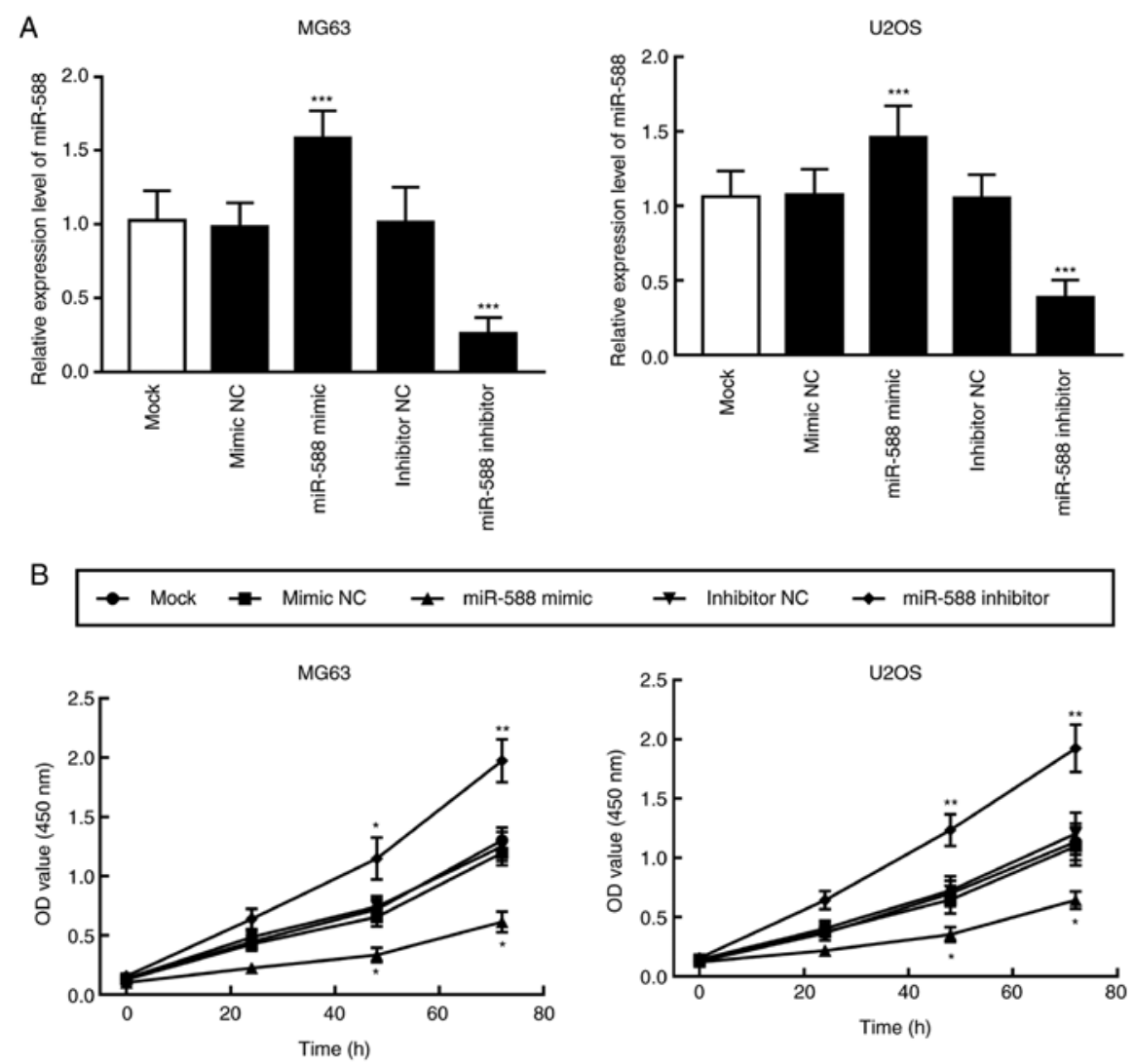

Figure 3. Effects of miR-588 expression level on OS cell proliferation. (A) Cell transfection with miR-588 mimic significantly increased the expression of miR-588 in MG63 and U2OS cells. Transfection of miR-588 inhibitor significantly decreased the expression of miR-588. ${ }^{* * *} \mathrm{P}<0.001$ vs. Mock group. (B) Proliferation of MG63 and U2OS cells was promoted by miR-588 knockdown and inhibited miR-588 overexpression. * $\mathrm{P}<0.05$ and ${ }^{* * *} \mathrm{P}<0.01$ vs. Mock group. OS, osteosarcoma; miR, microRNA; NC, negative control; OD, optical density.

performed to regulate the expression of miR-588 in MG63 and U2OS cells, which presented the lowest miR-588 expression (Fig. 1B). Compared with the Mock and NC groups, miR-588 expression in MG63 and U2OS cells transfected with miR-588 mimic was significantly increased, whereas its expression was significantly decreased in miR-588 inhibitor transfected cells $(\mathrm{P}<0.001$; Fig. 3A). Furthermore, the effect of transfections with miR-588 mimic and miR-588 inhibitor on OS cell proliferation was determined with the CCK-8 assay. The results demonstrated that miR-588 overexpression significantly inhibited the proliferation of MG63 and U2OS cells, whereas miR-588 knockdown significantly stimulated the proliferation of OS cells $(\mathrm{P}<0.01$ and $\mathrm{P}<0.05$; Fig. $3 \mathrm{~B})$.
miR-588 inhibits the migratory and invasive abilities of $O S$ cells. The migratory and invasive abilities of OS transfected cells were evaluated by Transwell assays. The results demonstrated that the number of migrated and invasive cells in the miR-588 mimic group was significantly decreased compared with that in the Mock and NC groups. Conversely, miR-588 knockdown significantly increased the number of migrated and invasive MG63 and U2OS cells ( $\mathrm{P}<0.001$; Fig. 4A and B).

\section{Discussion}

OS is a common bone tumor characterized by rapid development, high metastatic potential and poor prognosis $(24,25)$. 
A
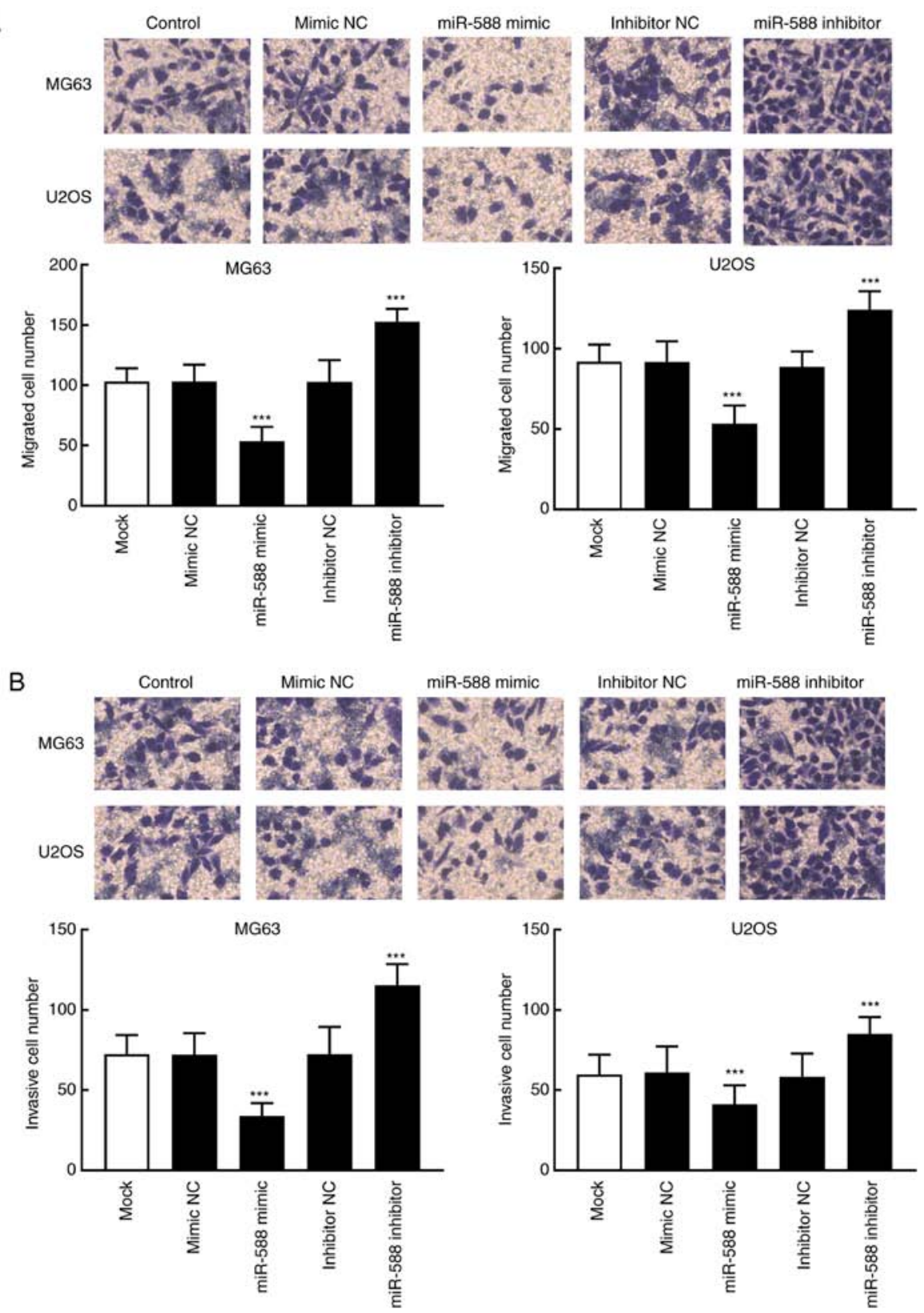

Figure 4. Effects of miR-588 expression level on the migratory and invasive abilities of OS cells. (A) Overexpression of miR-588 significantly decreased the migratory ability of MG63 and U2OS cells. Downregulation of miR-588 significantly increased the migratory ability of MG63 and U2OS cells. ${ }^{* * *} \mathrm{P}<0.001$ vs. Mock group. (B) Overexpression of miR-588 significantly inhibited the invasive ability of MG63 and U2OS cells. Downregulation of miR-588 significantly increased the invasive ability of MG63 and U2OS cells. ${ }^{* * *} \mathrm{P}<0.001$ vs. Mock group. OS, osteosarcoma; miR, microRNA; NC, negative control.

Novel efficient biomarkers are therefore required to improve the survival rate of patients with OS. Previous studies reported that miRNAs are involved in various cellular functions and biological processes, including cell proliferation, differentiation, apoptosis and invasion $(26,27)$. Therefore, determining the miRNAs involved in OS tumor formation might help the development of novel therapeutic strategies for the treatment and management of OS (28). It has been demonstrated that several miRNAs are dysregulated in OS and serve crucial roles in OS tumor progression. For example, it was reported that miR-139-5p expression is downregulated in OS tissues and cells and that miR-139-5p overexpression can significantly inhibit the progression of OS (29). It is therefore necessary to determine the function of dysregulated miRNAs in OS progression.
In prostate cancer, miR-588 was demonstrated to be upregulated, and its expression is associated with the poor clinical prognosis and survival rate of patients (19). However, the expression of miR-588 was different in OS. In the present study, the downregulation of miR-588 in OS was demonstrated to be associated with MSTS staging of patients with OS, which was consistent with the previously reported miRNA expression profile of OS (22). Similar findings in breast cancer showed that significant miR-588 downregulation could promote cancer cell proliferation and decrease cisplatin chemosensitivity (18). Furthermore, miR-588 downregulation also predicted the poor prognosis of patients with OS. In the present study, miR-588 expression and MSTS staging were demonstrated to act as independent 
prognostic factors for OS. The prognostic value of miR-588 was also previously demonstrated in breast and prostate cancers $(18,19)$. The prognostic value of miRNAs has drawn special attention in previous studies, and several miRNAs have been identified as independent prognostic indicators for various types of cancer (30). For instance, miR-1294 is downregulated in gastric cancer and its expression is associated with shorter disease-free survival and overall survival of patients. miR-1294 was also identified as an independent risk factor for gastric cancer prognosis by multivariate Cox analysis (31). The relatively small clinical sample size of the present study was a limitation, which might also limit the interpretation of the results. It is therefore necessary to conduct further investigation using a larger sample size.

Regarding the biological function of miR-588 in the development of OS, the present study demonstrated that miR-588 downregulation significantly stimulated OS cell proliferation and migratory and invasive abilities, whereas miR-588 overexpression had the opposite effect. Previously, miR-588 was also found to inhibit gastric tumor progression to its inhibitory effect on gastric cancer cell invasion and migration (20). Furthermore, miR-588 was reported to be downregulated in lung squamous cell carcinoma and to act as a tumor suppressor that could inhibit tumor cell migration and invasion by targeting progranulin (21). These results indicated that miR-588 may be involved in OS tumor development and that it may serve a tumor suppressor role in OS.

Although the expression and functional role of miR-588 in OS were confirmed in the present study, the underlying mechanism by which miR-588 regulates the progression and development of OS was not determined and requires therefore further investigation. In addition, in vivo studies are also necessary to confirm the results from in vitro studies (32). The present study provided in vitro evidence demonstrating the potential tumor suppressor role of miR-588 in the development of OS, which needs to be validated by in vivo experiments.

In conclusion, the present study demonstrated that miR-588 was downregulated in OS tissues and cells. In addition, miR-588 expression was associated with MSTS staging of patients with OS and miR-588 downregulation indicated a poor prognosis for these patients. Furthermore, the proliferation and migratory and invasive abilities of OS cells were stimulated following miR-588 downregulation and inhibited by miR-588 overexpression. Taken together, these findings suggested that miR-588 may be considered as a biomarker for the prognosis of OS and a tumor suppressor in the development of OS.

\section{Acknowledgements}

Not applicable.

\section{Funding}

No funding was received.

\section{Availability of data and materials}

The datasets used and/or analyzed during the current study are available from the corresponding author on reasonable request.

\section{Authors' contributions}

TY and SL analyzed and interpreted the data from patients. TY, SL, TM and WS performed the experiments and wrote manuscript. TY and WS confirm the authenticity of all the raw data. All authors have read and approved the final manuscript.

\section{Ethics approval and consent to participate}

This study was approved by the Ethics Committee of The Second Affiliated Hospital of Mudanjiang University (approval no. 200934; Mudanjiang, China). All patients signed written informed consent.

\section{Patient consent for publication}

Not applicable.

\section{Competing interests}

The authors declare that they have no competing interests.

\section{References}

1. Picci P: Osteosarcoma (osteogenic sarcoma). Orphanet J Rare Dis 2: 6, 2007.

2. Ottaviani $\mathrm{G}$ and Jaffe N: The epidemiology of osteosarcoma. Cancer Treat Res 152: 3-13, 2009.

3. Liu W, Zhang X, Liu P, Shen X, Lan T, Li W, Jiang Q, Xie X and Huang $\mathrm{H}$ : Effects of berberine on matrix accumulation and NF-kappa B signal pathway in alloxan-induced diabetic mice with renal injury. Eur J Pharmacol 638: 150-155, 2010.

4. Yoshida K, Okamoto M, Aoki K, Takahashi J and Saito N: A Review of T-Cell Related Therapy for Osteosarcoma. Int J Mol Sci 21: 4877, 2020.

5. Punzalan M and Hyden G: The role of physical therapy and occupational therapy in the rehabilitation of pediatric and adolescent patients with osteosarcoma. Cancer Treat Res 152: 367-384, 2009.

6. Daw NC, Chou AJ, Jaffe N, Rao BN, Billups CA, Rodriguez-Galindo C, Meyers PA and Huh WW: Recurrent osteosarcoma with a single pulmonary metastasis: A multi-institutional review. Br J Cancer 112: 278-282, 2015.

7. Meazza C and Scanagatta P: Metastatic osteosarcoma: A challenging multidisciplinary treatment. Expert Rev Anticancer Ther 16: 543-556, 2016.

8. Sun HH, Chen XY, Cui JQ, Zhou ZM and Guo KJ: Prognostic factors to survival of patients with chondroblastic osteosarcoma. Medicine (Baltimore) 97: e12636, 2018.

9. Imura Y, Takenaka S, Kakunaga S, Nakai T, Wakamatsu T, Outani H, Tanaka T, Tamiya H, Oshima K, Hamada K, et al: Survival analysis of elderly patients with osteosarcoma. Int Orthop 43: 1741-1747, 2019.

10. Liao YX, Yu HY, Lv JY, Cai YR, Liu F, He ZM and He SS: Targeting autophagy is a promising therapeutic strategy to overcome chemoresistance and reduce metastasis in osteosarcoma. Int J Oncol 55: 1213-1222, 2019.

11. Lagos-Quintana M, Rauhut R, Lendeckel W and Tuschl T: Identification of novel genes coding for small expressed RNAs. Science 294: 853-858, 2001.

12. Qiao W, Cao N and Yang L: MicroRNA-154 inhibits the growth and metastasis of gastric cancer cells by directly targeting MTDH. Oncol Lett 14: 3268-3274, 2017.

13. Yang ZQ, Wu CA and Cheng YX: Prognostic value of microRNA-133a expression and its clinicopathologic significance in non-small cell lung cancer: a comprehensive study based on meta-analysis and the TCGA database. Oncol Res Treat 41: 762-768, 2018.

14. Li C, Zhang J, Ma Z, Zhang F and Yu W: miR-19b serves as a prognostic biomarker of breast cancer and promotes tumor progression through PI3K/AKT signaling pathway. OncoTargets Ther 11: 4087-4095, 2018. 
15. Wang L, Xu M, Lu P and Zhou F: microRNA-769 is downregulated in colorectal cancer and inhibits cancer progression by directly targeting cyclin-dependent kinase 1 . OncoTargets Ther 11: 9013-9025, 2018.

16. Ji Q, Xu X, Song Q, Xu Y, Tai Y, Goodman SB, Bi W, Xu M, Jiao S, Maloney WJ, et al: miR-223-3p inhibits human osteosarcoma metastasis and progression by directly targeting CDH6. Mol Ther 26: 1299-1312, 2018.

17. Cai W, Xu Y, Yin J, Zuo W and Su Z: miR-552-5p facilitates osteosarcoma cell proliferation and metastasis by targeting WIF1. Exp Ther Med 17: 3781-3788, 2019.

18. Yu M, Zhang X, Li H, Zhang P and Dong W: MicroRNA-588 is downregulated and may have prognostic and functional roles in human breast cancer. Med Sci Monit 23: 5690-5696, 2017.

19. Zhao N, Lin T, Zhao C, Zhao S, Zhou S and Li Y: MicroRNA-588 is upregulated in human prostate cancer with prognostic and functional implications. J Cell Biochem: Oct 5, 2017 (Epub ahead of print).

20. Zhou X, Xu M, Guo Y, Ye L, Long L, Wang H, Tan P and $\mathrm{Xu}$ M: MicroRNA-588 regulates invasion, migration and epithelial-mesenchymal transition via targeting EIF5A2 pathway in gastric cancer. Cancer Manag Res 10: 5187-5197, 2018.

21. Qian L, Lin L, Du Y, Hao X, Zhao Y and Liu X: MicroRNA-588 suppresses tumor cell migration and invasion by targeting GRN in lung squamous cell carcinoma. Mol Med Rep 14: 3021-3028, 2016.

22. Chen G, Yu W, Li Z, Wang Q, Yang Q, Du Z, Zhang G and Song Y: Potential regulatory effects of miR-182-3p in osteosarcoma via targeting EBF2. BioMed Res Int 2019: 4897905, 2019.

23. Livak KJ and Schmittgen TD: Analysis of relative gene expression data using real-time quantitative PCR and the 2(-Delta Delta C(T)) Method. Methods 25: 402-408, 2001.
24. Wang SY, Hu HZ, Qing XC, Zhang ZC and Shao ZW: Recent advances of drug delivery nanocarriers in osteosarcoma treatment. J Cancer 11: 69-82, 2020.

25. Qiu S, Tao L and Zhu Y: Marital Status and Survival in Osteosarcoma Patients: An Analysis of the Surveillance, Epidemiology, and End Results (SEER) Database. Med Sci Monit 25: 8190-8203, 2019.

26. Bushati N and Cohen SM: microRNA functions. Annu Rev Cell Dev Biol 23: 175-205, 2007.

27. Mohr AM and Mott JL: Overview of microRNA biology. Semin Liver Dis 35: 3-11, 2015.

28. Wang J, Liu S, Shi J, Li J, Wang S, Liu H, Zhao S, Duan K, Pan X and Yi Z: The Role of miRNA in the Diagnosis, Prognosis, and Treatment of Osteosarcoma. Cancer Biother Radiopharm 34: 605-613, 2019.

29. Shi YK and Guo YH: MiR-139-5p suppresses osteosarcoma cell growth and invasion through regulating DNMT1. Biochem Biophys Res Commun 503: 459-466, 2018.

30. Iorio MV and Croce CM: MicroRNA dysregulation in cancer: Diagnostics, monitoring and therapeutics. A comprehensive review. EMBO Mol Med 4: 143-159, 2012.

31. Shi YX, Ye BL, Hu BR and Ruan XJ: Expression of miR-1294 is downregulated and predicts a poor prognosis in gastric cancer. Eur Rev Med Pharmacol Sci 22: 5525-5530, 2018.

32. Chen Y, Gao DY and Huang L: In vivo delivery of miRNAs for cancer therapy: Challenges and strategies. Adv Drug Deliv Rev 81: 128-141, 2015. 